\title{
Theoretical Status of Neutrino Oscillation Physics
}

\author{
Sin Kyu Kang* \\ School of Liberal Arts, Seoul Tech, Gongreung-ro 232, Nowon-gu, Seoul 01811, Korea \\ E-mail: skkangeseoultech.ac.kr
}

\begin{abstract}
We present recent results of the global analysis of neutrino oscillation data including LBL data from $\mathrm{T} 2 \mathrm{~K}$ and NOvA, SBL results from the reactor experiments Daya Bay and RENO, and the atmospheric neutrino results from Super-Kamiokande have been included in the fit, and discuss how precisely neutrino oscillation parameters are determined. The current status of determinations of neutrino mass ordering, $\mathrm{CP}$ violation and octant of $\theta_{23}$ from the fit by using latest neutrino data are discussed. We also discuss recent developments on the origin of the neutrino mixing pattern, and how such a tiny neutrino mass can be generated. Several recent proposals for neutrino mixing patterns and tiny neutrino masses are introduced. Recent developments on the impact of non-standard interactions and non-unitarity of neutrino mixing matrix on the results for neutrino oscillations are addressed.
\end{abstract}

The 21st international workshop on neutrinos from accelerators (NuFact2019)

August 26 - August 31, 2019

Daegu, Korea

${ }^{*}$ Speaker. 


\section{Introduction}

Neutrinos are the most mysterious and fascinating of all elementary particles puzzling physicists. It is common belief that they play a very important role in both of the microscopic view of particle physics and the macroscopic view of evolution of the universe. The remarkable development over the past two decades has been the discovery of the flavor oscillations in neutrinos[1, 2, 3, 4, 5], which has established that neutrinos have masses and mix.

In the framework of three active neutrinos, neutrino oscillations can be described by 6 independent parameters, of which three mixing angles and two mass squared differences, have been measured by the experiments using neutrinos coming from the Sun, atmosphere, reactor and accelerators $[1,2,3,4,5]$. The remaining parameter in the mixing matrix, $\mathrm{CP}$ violating phase, becomes the main target of next generation of neutrino experiments [6]. Although neutrinos turned out to be massive particle, their individual masses have not yet been measured. The hierarchy of their masses is also one of the big mysteries, as the ordering of their values has profound implication in particle physics and cosmology [7, 8].

On the other hand, there are other issues that keep neutrino physicists puzzled, which are known to be "neutrino anomalies" that are unexpected results in several experimental studies [9]. They challenge the standard explanation of neutrino oscillations which have been used to determine three mixing angles and two mass squared differences in the framework of three active neutrinos.

In this talk, we briefly review on the recent development of neutrino physics from the theoretical perspective. Starting from presenting the current experimental status of neutrino mixing and masses, we discuss how we can understand the pattern of neutrino mixing and how tiny neutrino masses can naturally be generated. We study the issue on leptonic CP violation, and present how we can predict Dirac CP phase. Finally, we discuss the effects of non-standard interactions and non-unitarity of neutrino mixing matrix on the interpretation of neutrino data in terms of neutrino oscillation.

\section{Status of neutrino mixing and masses}

Current neutrino oscillation data obtained from the various neutrino sources coming from the Sun, atmosphere, reactors and accelerators can be interpreted in the framework of 3-neutrino paradigm, where the flavor eigenstates $v_{\alpha}=\left(v_{e}, v_{\mu}, v_{\tau}\right)$ are admixtures of the three mass eigenstates $v_{i}=\left(v_{1}, v_{2}, v_{3}\right)$ through a unitary mixing matrix depending on three mixing angles $\left(\theta_{12}, \theta_{23}, \theta_{13}\right)$, one possible Dirac CP-violating phase $\delta$ and two additional phases if neutrinos are Majorana particles [10]. Note that the standard parametrization presented in [10] is adopted in this presentation. Neutrino oscillations are driven by the two mass squared differences, $\Delta m_{21}^{2} \equiv m_{2}^{2}-m_{1}^{2},\left|\Delta m_{31}^{2}\right| \equiv$ $\left|m_{3}^{2}-m_{1}^{2}\right|$. The two cases $\Delta m_{31}^{2}>0$ and $\Delta m_{31}^{2}<0$ correspond to normal (NO) and inverted (IO) neutrino mass ordering. Thanks to the global fit to neutrino data, we could determine five oscillation parameters, the three mixing angles, two mass squared differences with percent accuracy, while the octant of $\theta_{23}$, the sign of $\Delta m_{31}^{2}$ and the phase $\delta$ are still unknown. Fig. 1 presents how precisely $\theta_{12}, \theta_{13}$ and $\Delta m_{21}^{2}, \Delta m_{31}^{2}$ are measured, whereas how less precisely $\theta_{23}$ and CP phase $\delta$ as well as mass ordering.

The constraints on $\left(\Delta m_{21}^{2}, \theta_{12}\right)$ come from the fit to the solar and KamLAND (Solar + KL) data, 

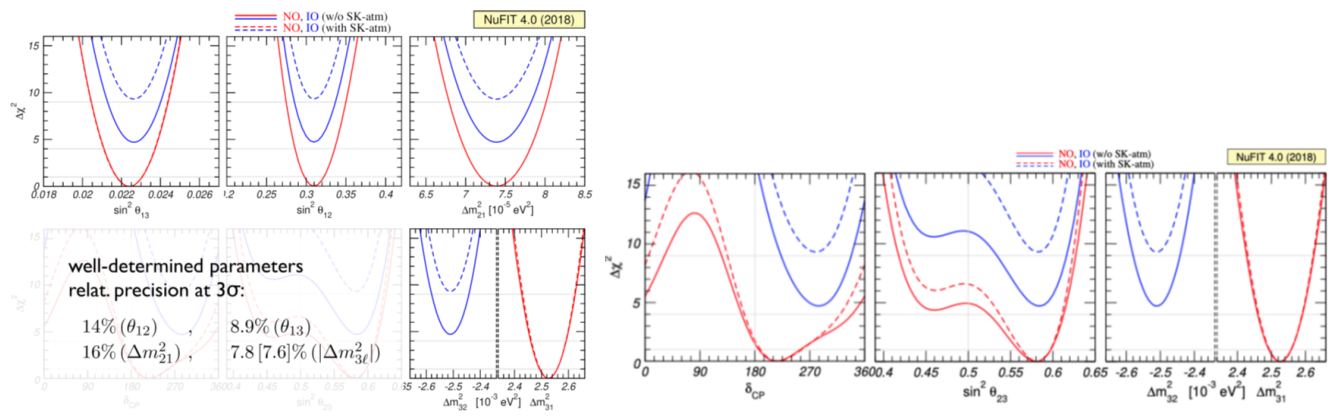

Figure 1: $\delta \chi^{2} v s$. neutrino oscillation parameters.

whereas those on $\left(\left|\Delta m_{31}^{2}\right|, \theta_{23}\right)$ from the fit to the atmospheric and accelerator data. Subsequently, long baseline (LBL)+Solar+KL data combined with short baseline reactor data (SBL Reactors) can precisely determine $\theta_{13}$ and give bounds on $\Delta m_{31}^{2}$ starting to be comparable with those coming from LBL and atmospheric neutrino data. The synergy between LBL+Solar+KL data and SBL Reactor data significantly improves the sensitivity to $\delta$ [11]. We obtain the allowed parameter ranges at $N_{\sigma}$,with $N_{\sigma}^{2}=\chi^{2}-\chi_{\min }^{2}[10]$ depending on the oscillation parameters $\left(\Delta m_{21}^{2},\left|\Delta m_{31}^{2}\right|, \theta_{12}, \theta_{13}, \theta_{23}\right)$ and on a certain number of systematic parameters [11, 12, 13]. For the details of the analysis and the input data sets used see $[11,12]$. While the Solar, KamLAND, MINOS and DeepCore IceCube data are unchanged with respect to [11], the analysis of the T2K and NOvA experiments, both in appearance and disappearance channels, the atmospheric neutrino data analysis by including the latest Super-Kamiokande data (phase I-IV, 0.33 Mtyr exposure) and SBL analysis by using the latest Daya Bay [3] and RENO [4] data are updated. The results of global fit to all the available neutrino oscillation data are summarized in Table 1, where the best-fit values and the allowed 1 and $3 \sigma$ ranges are reported, for both mass orderings with relative $1 \sigma$ uncertainty [14]. We note that the NO is favored over the IO, with $\delta \chi^{2}=\chi_{I O}^{2}-\chi_{N O}^{2}=3.5$.

\begin{tabular}{l|c|c|c}
\hline parameter & best fit $\pm 1 \sigma$ & $3 \sigma$ range & \\
\hline$\Delta m_{21}^{2}\left[10^{-5} \mathrm{eV}^{2}\right]$ & $7.55_{-0.16}^{+0.20}$ & $7.05-8.14$ & $2.4 \%$ \\
$\Delta m_{31}^{2}\left[10^{-3} \mathrm{eV}^{2}\right](\mathrm{NO})$ & $2.50 \pm 0.03$ & $2.41-2.60$ & $1.3 \%$ \\
$\Delta m_{31}^{2}\left[10^{-3} \mathrm{eV}^{2}\right](\mathrm{IO})$ & $2.42_{-0.04}^{+0.03}$ & $2.31-2.51$ & $1.3 \%$ \\
$\sin ^{2} \theta_{12} / 10^{-1}$ & $3.20_{-0.16}^{+0.04}$ & $2.73-3.79$ & $5.5 \%$ \\
$\sin ^{2} \theta_{23} / 10^{-1}(\mathrm{NO})$ & $5.47_{-0.30}^{+0.20}$ & $4.45-5.99$ & $4.7 \%$ \\
$\sin ^{2} \theta_{23} / 10^{-1}(\mathrm{IO})$ & $5.51_{-0.30}^{+0.18}$ & $4.53-5.98$ & $4.4 \%$ \\
$\sin ^{2} \theta_{13} / 10^{-2}(\mathrm{NO})$ & $2.160_{-0.069}^{+0.083}$ & $1.96-2.41$ & $3.5 \%$ \\
$\sin ^{2} \theta_{13} / 10^{-2}(\mathrm{IO})$ & $2.220_{-0.076}^{+0.074}$ & $1.99-2.44$ & $3.5 \%$ \\
$\delta / \pi(\mathrm{NO})$ & $1.32_{-0.15}^{+0.21}$ & $0.87-1.94$ & $10 \%$ \\
$\delta / \pi(\mathrm{IO})$ & $1.56_{-0.15}^{+0.13}$ & $1.12-1.94$ & $9 \%$ \\
\hline
\end{tabular}

Table 1: Results of global fit to neutrino data for neutrino oscillation parameters. The 4th column represents relative $1 \sigma$ uncertainty. 


\section{Theoretical Issues}

\subsection{Origin of neutrino mixing pattern and $\mathrm{CP}$ phase}

From fit to neutrino data in 3-neutrino paradigm, neutrino mixing matrix looks much different from quark mixing matrix. A natural question concernd with neutrino mixing patterns is how we can understand neutrino mixing matrix from the theoretical perspective. Before measuring $\theta_{13}$, the so-called tri-bimaximal mixing (TBM) hypothesis given by

$$
U_{\mathrm{TBM}}=\left(\begin{array}{ccc}
\frac{2}{\sqrt{6}} & -\frac{1}{\sqrt{3}} & 0 \\
\frac{1}{\sqrt{6}} & \frac{1}{\sqrt{3}} & -\frac{1}{\sqrt{2}} \\
\frac{1}{\sqrt{6}} & \frac{1}{\sqrt{3}} & \frac{1}{\sqrt{2}}
\end{array}\right)
$$

has been accepted and studied how the TBM can be generated.

In fact, the TBM matrix can diagonalize the neutrino mass matrix as follows [16];

$$
\begin{aligned}
U_{\mathrm{TBM}} M_{v}^{D} U_{\mathrm{TBM}}^{T} & =\left(\begin{array}{ccc}
m_{1} & m_{2} & m_{2} \\
m_{2} & \frac{1}{2}\left(m_{1}+m_{2}+m_{3}\right) & \frac{1}{2}\left(m_{1}+m_{2}-m_{3}\right) \\
m_{2} & \frac{1}{2}\left(m_{1}+m_{2}-m_{3}\right) & \frac{1}{2}\left(m_{1}+m_{2}+m_{3}\right)
\end{array}\right) \\
& =\frac{m_{1}+m_{3}}{2}\left(\begin{array}{lll}
1 & 0 & 0 \\
0 & 1 & 0 \\
0 & 0 & 1
\end{array}\right)+\frac{m_{2}-m_{1}}{3}\left(\begin{array}{lll}
1 & 1 & 1 \\
1 & 1 & 1 \\
1 & 1 & 1
\end{array}\right)+\frac{m_{1}-m_{3}}{2}\left(\begin{array}{lll}
1 & 0 & 0 \\
0 & 0 & 1 \\
0 & 1 & 0
\end{array}\right)
\end{aligned}
$$

In the above expression, the integer matrix elements suggest non-Abelian discrete symmetry. There are a lot of non-Abelian discrete symmetries can generate the TBM matrix [17]. But, the exact TBM mixing pattern is ruled out by the results from react neutrino experiments $[3,4,5]$; thus, we need to modify it to have non-zero $(1,3)$ component. If the deviation of neutrino mixing matrix from the TBM form is not substantial, we can regard $U_{\text {TBM }}$ as a leading-order approximation. Because the non-trivial $(1,3)$ component may contain a CP phase, incorporating $\delta$ on modifying $U_{\text {TBM }}$ would be desirable. The minimal modification is to multiply $U_{\mathrm{TBM}}$ by a rotation unitary matrix in the (i,j) plane with an angle $\theta$ and a CP phase $\xi, U_{i j}(\theta, \xi)$ [18]. Then, the mixing angle $\theta$ introduced possibly gives rise to non-zero mixing angle $\theta_{13}$ and deviation from maximal mixing $\theta_{23}$. In Ref. 11, it is shown that the minimal modification of $U_{\text {TBM }}$ can be realized in a neutrino model with $A_{4}$ flavor symmetry by incorporating $A_{4}$ symmetry-breaking terms appropriately. Confronting those forms with experimental data, it turns out that the best fit is achieved by $U_{\mathrm{TBM}} U_{23}(\theta, \xi)$ among possible forms of the minimal modification. Following the procedures presented in [18] based on $U_{\mathrm{TBM}} U_{23}(\theta, \xi)$, one can obtain the relation between the solar and reactor mixing angles, and the relation between $\delta$ and neutrino mixing angles,

$$
s_{12}^{2}=\frac{1}{3\left(1-s_{13}^{2}\right)}, \quad \cos \delta=\frac{2-4 s_{13}^{2}}{s_{13} \sqrt{2-3 s_{13}^{2}}} .
$$

The relation (3.4) reflects that the CP phase $\delta$ can be predicted in terms of two neutrino mixing angles. In the left panel of Fig. 2, we plot $\delta$ along with $\theta_{23}$. Alternatively, the authors in [19] study a way to predict $\delta$ in terms of neutrino mixing angle $\theta_{23}$ as shown in right panel of Fig. 2. 

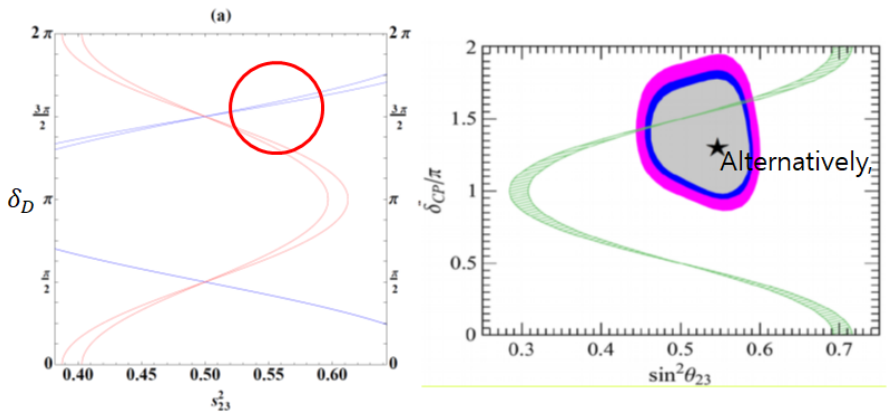

Figure 2: Predictions of $\delta$ in terms of $\theta_{23}$.

\subsection{Origin of neutrino masses}

As we know that neutrinos are massive particle, it is necessary to modify the SM Lagrangian including the neutrino mass terms and then to account for the origin of them in a gauge invariant manner. Since there are no right-handed neutrinos, we can not add a Dirac neutrino mass terms. Majorana mass terms can be constructed using the $v_{L}$ fields only, but breaks the SM gauge invariance. Consequently, the SM does not allow for neutrino masses without introducing new heavy particles and higher dimensional effective terms. In this sense, neutrino masses and mixing serve as the evidence that the SM is incomplete. Therefore, we are forced to construct neutrino mass terms in a consistent framework. In addition, we should invent a mechanism to explain why neutrino masses are so small. A vast number of models has been proposed.

The see-saw type I mechanism is one of the simplest extensions of the SM which can explain not only neutrino masses but also their smallness. It breaks lepton number by two units and predicts Majorana neutrinos. Among all SM fermions, neutrinos are the only ones that can have a Majorana mass term. Noticing that the term $\bar{L} \cdot \tilde{H}$ is gauge invariant, it is possible to construct a singlet combination,

$$
\frac{y}{\Lambda} L^{T} \cdot \tilde{H}^{*} C^{\dagger} \tilde{H}^{\dagger} \cdot L+h . c .,
$$

where $C$ is charge conjugation operator, and $\Lambda$ is a mass scale. This term, called the Weinberg operator, has dimension 5 [20]. After Higgs field gets a vacuum expectation value, the Weinberg operator leads to a Majorana mass term that breaks lepton number by two units,

$$
\frac{y v_{H}^{2}}{2 \Lambda} v_{L}^{T} C^{\dagger} v_{L}+\text { h.c.. }
$$

Since the Weinberg operator of Eq. (3.5) is a $(d=5)$ operator, it implies that the SM is the low energy remnant of a higher energy theory. It should be effectively generated by integrating out some new heavy particles contained in a higher energy theory. At tree level, there exist only three different realizations of the Weinberg operator, which are characterized by the quantum numbers of the new mediators Fig. 3 shows how tiny neutrino masses can be generated at tree level via seesaw mehcanisms. [21],

- Type-I Seesaw: mediated by heavy Majorana right-handed neutrinos $N_{R}$ that are singlets under the SM gauge group. 
- Type-II Seesaw: mediated by heavy $S U(2)_{L}$ triplets of scalar fields $\Phi$ with hypercharge $Y=2$

- Type-III Seesaw: mediated by heavy $S U(2)_{L}$ fermionic triplets $\Sigma_{R}$ with zero hypercharge.

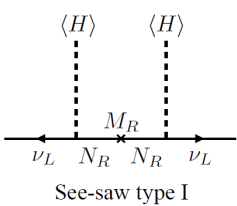

See-saw type I

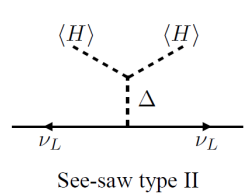

See-saw type II
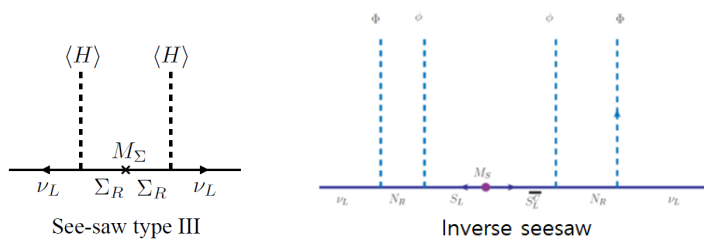

Figure 3: Diagrams for Seesaw and inverse seesaw mechanisms

Alternatively, tiny neutrino masses can be generated though the so-called inverse seesaw mechanism [22]. It works at a much lower scale than seesaw mechanisms shown above. It is also realized with rather large Yukawa couplings. The model leading to inverse seesaw contains a very small lepton number violating parameter, $\mu_{S} \sim \mathrm{eV}$, which is crucial to achieve the smallness of the light neutrino masses. Thanks to the presence of $\mu_{S}$, the Yukawa coupling generating the Dirac neutrino mass term can be quite large $(\sim 0.1)$ even in the presence of sub $\mathrm{TeV}$ heavy neutrino masses in this scenario. One of advantages of inverse seesaw is testable at colliders. Phenomenology of inverse seesaw mechanism and its variants such as minimal inverse seesaw, littlest inverse seesaw and scotogenic inverse seesaw have been studied. Recently, several authors proposed alternatives to achieve naturally light Dirac neutrinos with masses induced via seesaw mechanism [23]. Similar to the seesaw mechanism for Majorana neutrinos, there exist type-I,II, III seesaw for Dirac neutrinos.

Tiny neutrino masses can also be generated at loop level [24]. In the radiative neutrino mass models, new scalar fields are added on top pf standard model (SM), and matrices of their Yukawa couplings determine the structure of the neutrino mass matrix. There are variants of the radiative neutrino mass models as shown in Fig. 4.

\subsection{New physics in neutrino oscillations}

\subsubsection{Non-standard Neutrino interactions}

Non-standard neutrino interactions(NSIs) provide a general framework of effective field theory to quantify new physics in the neutrino sector1. NSIs are typically presented in the following forms of neutral current (NC) and charged current(CC) interactions [25],

$$
\begin{aligned}
& \mathscr{L}_{N C}=-2 \sqrt{2} G_{F} \sum_{f, P, \alpha, \beta} \varepsilon_{\alpha \beta}^{f, P}\left(\bar{v}_{\alpha} \gamma^{\mu} P_{L} v_{\beta}\right)\left(\bar{f} \gamma^{\mu} P f\right), \\
& \mathscr{L}_{C C}=-2 \sqrt{2} G_{F} \sum_{f, P, \alpha, \beta} \varepsilon_{\alpha \beta}^{f, P}\left(\bar{v}_{\alpha} \gamma^{\mu} P_{L} l_{\beta}\right)\left(\bar{f} \gamma^{\mu} P f^{\prime}\right),
\end{aligned}
$$

where the sum is over fermions such as $f, f^{\prime} \in\{e, u, d\}, P \in\left\{P_{L}, P_{R}\right\}$ are the chirality projection operators, $G_{F}$ is Fermi's constant and the $\varepsilon$ terms quantify the size of the new interaction 

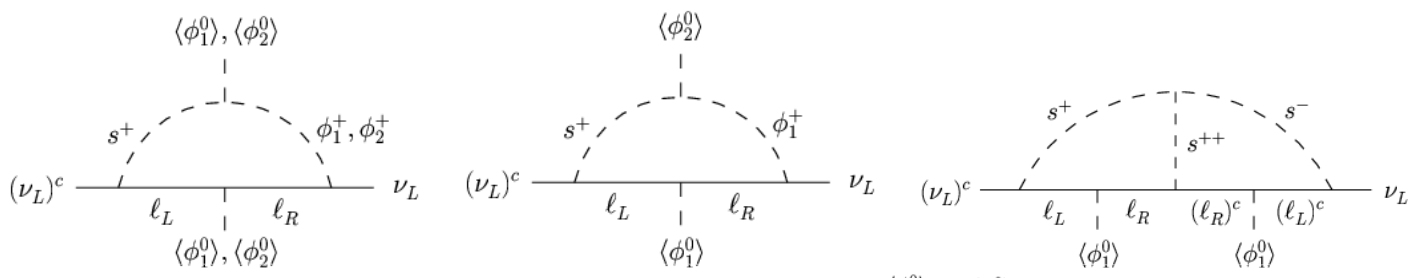

$\left\langle\phi_{1}^{0}\right\rangle \quad\left\langle\phi_{1}^{0}\right\rangle$
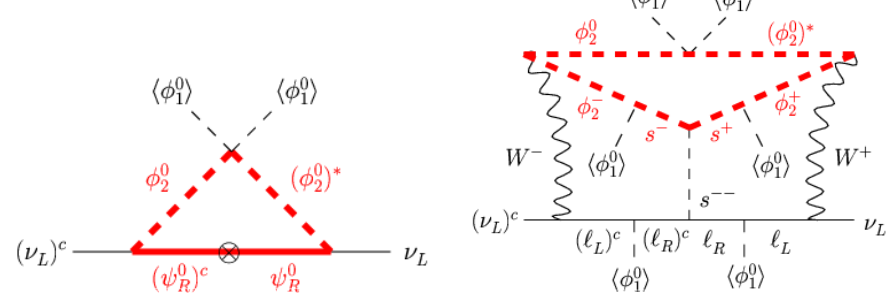

Figure 4: Diagrams for radiative generation of neutrino masses.

relative to the weak scale. NSI generally leads to a rich phenomenology in both scattering experiments and neutrino oscillation experiments [26]. Since oscillation phenomenology is generally quite distinct from scattering phenomenology, the NSI can provides a convenient way to relate new physics to both cases.

The effect of NSI in propagation can be presented through the modification of matter potential given as [27]

$$
H_{\text {matt }}=\sqrt{2} G_{F} N_{e}(x)\left(\begin{array}{ccc}
1+\varepsilon_{e e}(x) & \varepsilon_{e \mu}(x) & \varepsilon_{e \tau}(x) \\
\varepsilon_{e \mu}^{*}(x) & \varepsilon_{\mu \mu}(x) & \varepsilon_{\mu \tau}(x) \\
\varepsilon_{e \tau}^{*}(x) & \varepsilon_{\mu \tau}^{*}(x) & \varepsilon_{\tau \tau}(x)
\end{array}\right)
$$

It is worthwhile to notice that transition $v_{\alpha} \rightarrow v_{\beta}$ can occur via the interactions even if there exists no mixing in vacuum. The comple phases of off-diagonal component of $H_{\text {matt }}$ could be a new source of $\mathrm{CP}$ violation. The current conservative model independent bounds are given as

$$
\left(\begin{array}{rr}
\left|\varepsilon_{e e}\right|<4.2\left|\varepsilon_{e \mu}\right|<0.33 & \left|\varepsilon_{e \tau}\right|<3.0 \\
\left|\varepsilon_{\mu \mu}\right|<0.07 & \left|\varepsilon_{\mu \tau}\right|<0.33 \\
& \left|\varepsilon_{\tau \tau}\right|<21
\end{array}\right) .
$$

NSI may affect neutrinos at the production point as well as detection point. To see those effects, we use different parameters. The bounds on those effects coming from global fit to solar data and KamLAND data are presented in [27].

It is worthwhile to notice that NSI may prevent determination of CP violation coming from neutrino mixing matrix mainly because the parameters associated with NSI contains unknown CP phases. Fig. 5 shows how uncertainties for the determination of $\mathrm{CP}$ phase in neutrino mixing matrix can be increased along with the values of $\left|\varepsilon_{\alpha \beta}\right|$ [28]. There are lots of the origins of NSI coming from integrating out heavy new particles, loop effects and higher dimensional operators. 


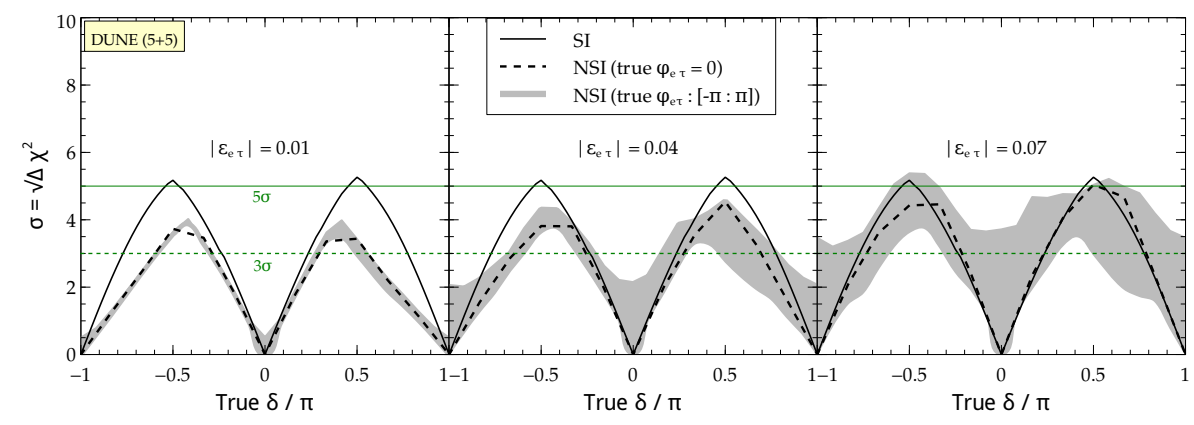

Figure 5: $\delta$ vs. $\sigma$ for three inputs of $\varepsilon_{\alpha \beta}$. Shaded regions represent uncertainties to determine CP phase.

\subsubsection{Non-unitarity of neutrino mixing matrix}

There are a lot of sources of non-unitarity of neutrino mixing matrix such as the existence of sterile neutrino, effective operators and minimal unitarity violation. One of popular parametrization for the non-unitarity is [29]

$$
N=N^{N P} U_{\mathrm{PMNS}}=\left(\begin{array}{ccc}
\alpha_{11} & 0 & 0 \\
\alpha_{21} & \alpha_{22} & 0 \\
\alpha_{31} & \alpha_{32} & \alpha_{33}
\end{array}\right)
$$

The parameters $\alpha_{i j}$ are constrained from various experiments such as neutrino oscillation, $W$ and $Z$ boson decays, rare lepton flavor violating decays, lepton universality tests, etc. The current bounds are presented in [30]. The sensitivity to $\mathrm{CP}$ violation in long baseline experiments can be affected by the presence of non-unitarity.

\section{4. conclusion}

In this talk we have presented recent results of the global analysis of neutrino oscillation data including LBL data from T2K and NOvA, SBL results from the reactor experiments Daya Bay and RENO, and the atmospheric neutrino results from Super-Kamiokande have been included in the fit. As a result of the global fit, three neutrino mixing angles and two mass squared difference have been determined with percent fractional accuracies of $(2.4 \%, 5.8 \%, 1.6 \%, 3.9 \%, 9 \%)$, respectively. We have discussed the current status of determinations of neutrino mass ordering, $\mathrm{CP}$ violation and octant of $\theta_{23}$ from the fit by using latest neutrino data. The current results show preference of NO in the first octant, IO in the second one, and a more pronounced preference for the normal mass order at a level of $\sim 1.9 \sigma$. We have discussed how we can understand the neutrino mixing pattern from theoretical perspective, and how such a tiny neutrino mass can be generated. Several recent proposals for neutrino mixing patterns and tiny neutrino masses have been introduced. We have presented recent development of the impact of non-standard interactions and non-unitarity of neutrino mixing matrix on the results for neutrino oscillations. 


\section{References}

[1] Y. Fukuda et al. [Super-Kamiokande Collaboration], Phys. Rev. Lett. 81 (1998) 1562.

[2] Q. R. Ahmad et al. [SNO Collaboration], Phys. Rev. Lett. 87 (2001) 071301.

[3] F. P. An et al. [Daya Bay Collaboration], Phys. Rev. Lett. 108 (2012) 171803.

[4] J. K. Ahn et al. [RENO Collaboration], Phys. Rev. Lett. 108 (2012) 191802.

[5] Y. Abe et al. [Double Chooz Collaboration], Phys. Rev. Lett. 108 (2012) 131801.

[6] H. Nunokawa, S. J. Parke and J. W. F. Valle, Prog. Part. Nucl. Phys. 60 (2008) 338; G. C. Branco, R. G. Felipe and F. R. Joaquim, Rev. Mod. Phys. 84 (2012) 151; S. F. King, Prog. Part. Nucl. Phys. 94 (2017) 217; S. T. Petcov, Eur. Phys. J. C 78 (2018) 709.

[7] S. Vagnozzi et al., Phys. Rev. D 96 (2017) 123503.

[8] R. B. Patterson, Ann. Rev. Nucl. Part. Sci. 65 (2015) 177.

[9] For a recent review, see C. Giunti and T. Lasserre, arXiv:1901.08330 [hep-ph]; S. K. Kang, Int. J. Mod. Phys. A 34 (2019) 1930005 (2019).

[10] M. Tanabashi et al. [Particle Data Group], Phys. Rev. D 98 (2018) 030001.

[11] F. Capozzi et al., Nucl. Phys. B 908 (2016) 218.

[12] F. Capozzi et al., Phys. Rev. D89 (2014) 093018.

[13] G. L. Fogli, E. Lisi, A. Marrone, D. Montanino and A. Palazzo, Phys. Rev. D66 (2002) 053010.

[14] P. F. de Salas et al., Phys. Lett. B 782 (2018) 633.

[15] P. F. Harrison, D. H. Perkins, and W. G. Scott, Phys. Lett. B530 (2002) 167.

[16] M. Tanimoto, See also S. Chang, S. K. Kang and K. Siyeon, Phys. Lett. B 597 (2004) 78.

[17] G. Altarelli and F. Feruglio, Rev. Mod. Phys. 82 (2010) 2701; H. Ishimori, T. Kobayashi, H. Ohki, Y. Shimizu, H. Okada and M. Tanimoto, Prog. Theor. Phys. Suppl. 183 (2010) 1.

[18] S. K. Kang, C. S. Kim, Phys. Rev. D 90 (2014) 077301; S. K. Kang and M. Tanimoto, Phys. Rev. D 91 (2015) 073010; ; S. K. Kang, J. Korean Phys. Soc. 71 (2017) 911.

[19] P. Chen et al., Phys. Rev. D 98 (2018) 055019.

[20] S. Weinberg, Phys.Rev.Lett. 43 (1979) 1566.

[21] For a review, see S. Pascoli, CERN Yellow Rep. School Proc. 6 (2019) 213. doi:10.23730/CYRSP-2019-006.213, and references therein.

[22] R. N. Mohapatra, Phys.Rev.,Lett. 56 (1986) 561; R. N. Mohapatra, J. Valle, Phys.Rev.D34 (1986) 1642.

[23] S. Centelles et al., Phys. Lett. B 767 (2017) 209;S. Chuliá et al., Phys. Lett. B 781 (2018) 122.

[24] For a review, H. Sugiyama, arXiv:1505.01738 and references therein.

[25] T. Ohlsseon, Rept.Prog.Phys.76 (2013) 044201; O.G. Miranda, H. Nunokawa, New. J. Phys. 17 (2015) 095002.

[26] V. D. Barger, R. J. N. Phillips, K. Whisnant, Phys. Rev. D44 (1991) 1629; M. M. Guzzo, A. Masiero, S. T. Petcov, Phys. Lett. B260 (1991) 154; Y. Grossman, Phys.Lett. B359 (1995) 141.

[27] I. Esteban et al., JHEP 1808 (2018) 180.

[28] M. Masud and P. Mehta, Phys. Rev. D 94 (2016) 013014.

[29] Z. z. Xing, Phys. Lett. B 660 (2008) 515 ; F. J. Escrihuela et al., Phys. Rev. D 92 (2015) 053009.

[30] F. J. Escrihuela et al., New J. Phys. 19 (2017) 093005. 